\title{
The 4C/ID-Model in Physics Education: Instructional Design of a Digital Learning Environment to Teach Electrical Circuits
}

\author{
Mário Melo \\ Instituto de Educação da Universidade de Lisboa, Portugal, mmlmelo@hotmail.com
}

$\mid$

In this paper, readers are guided through the design and development of educational programs based on the 4C/ID model. This was illustrated via a practical example in Physics education, to teach the theme "Electrical circuits" to students of the 9th grade of compulsory education. In the article, the followed steps are described, from prototyping to development and implementation, to building two learning modules: electrical circuits' representations, and characteristics of series, and parallel circuits. Furthermore, the study was to present an educational innovation that represents the transition from traditional, fragmented and classroom-based learning to an integrated digital learning environment based on experimental tested instructional design principles, to instructional designers and teachers. To test the effect of the learning environment on the students' learning, an experimental group was established on which the learning environment was used and a control group on which the theme of electrical circuits was taught by resorting to the teacher's exposition and performing exercises in the student's book.

Keywords: instructional design, the 4C/ID-model, physics education, electrical circuits, digital learning environment

\section{INTRODUCTION}

This paper describes the development of a digital learning environment to teach complex skills, in this case, the study of electrical circuits to $9^{\text {th }}$ grade Physics' students. The focus of this paper is on instructional design. It was also the objective of this study to investigate the effects of the instructional approach (4C/ID versus conventional) on the students' learning, in particular on the students' knowledge reproduction and learning transfer.

\section{THEORETICAL BACKGROUND}

\section{Instructional design}

Instructional design (ID) is defined as a systematic process that is employed to develop education and training programs in a consistent and reliable fashion (Reiser \& Dempsey, 2007). So, ID principles may provide a framework for developing more efficient

Citation: Melo, M. (2018). The 4C/ID-Model in Physics Education: Instructional Design of a Digital Learning Environment to Teach Electrical Circuits. International Journal of Instruction, 11(1), 103122. https://doi.org/10.12973/iji.2018.1118a 
educational training programs (Merrill et al., 1996). ID models typically specify a method that is followed to facilitate the knowledge transfer, skills and attitudes in new situations. The aim of ID is to facilitate the acquisition of knowledge in long-term memory via working memory that is limited in capacity and duration until it is transformed by knowledge held on long-term memory. Most of the current instructional models use multimedia features. Therefore, it is necessary to know how people process this kind of information.

The learning theories on which the 4C/ID-model is based are Sweller's Cognitive Load Theory and Mayer's Theory of Multimedia Learning. According to van Merrienböer and Kester (2014), the general (multimedia) cognitive learning theories can be categorized in three levels: (a) The first level corresponds to the psychological theories that describe the memory systems and the cognitive processes involved in learning (information processing theory). This theory is based on the human cognitive architecture, which comprises a working memory that interacts with a long-term memory. In this interaction the working memory queries the information retained in the long-term memory and if learning occurred the new information is recorded in mental schemas in the long-term memory, this process is called active processing by Mayer (Paas, et al., 2003); in the second level the theories for the design of educational messages that identify the multimedia principles and establish directions to create multimedia messages, in which the Sweller Cognitive Load Theory (Sweller et al., 2011) and the Mayer Multimedia Learning Theory (Mayer, 2014) are included; in the third level, the theories and models that prescribe ways to develop educational programs with the 4C/ID-model are included.

The specific contribution of the Theory of Cognitive Load was to establish a set of procedures, experimentally tested, to which instruction must obey in order to make learning more efficient. These procedures reduce the cognitive load from the working memory in order to facilitate the construction and/or improvement of mental schemas stored in long-term memory (Kirschner, 2002). Thus, according to this view, learning process corresponds to the construction of increasingly complex mental schemas that allows the release of the working memory during the learning process. Some examples of these principles that allow the reduction of the cognitive load imposed by the instructive material are: (i) 'The Goal-Free Effect'; (ii) 'The Worked Examples and Problem Completion Effect'; (iii) 'The Split-Attention Effect'; (iv) 'The Modality Effect' (Sweller, Ayres \& Kalyuga, 2011).

The Theory of Multimedia Learning has become very useful for those who intend to design digital educational resources. This theory assumes that people learn better when they associate words and images than when they use only words. This association must be made taking into account the human cognitive system. According to Mayer (2014), the memory is a cognitive system that allows the learning, i.e., the learned information is recorded in long-term memory serving as a support to new learning and/or to restructuring knowledge previously learned. Apart from the working memory and longterm memory, Mayer's theory predicts the existence of a sensory memory. Thus, Mayer's theory takes into account three assumptions: 1) the limited capacity of the working memory; 2) the fact that the human system of information processing has 
different channels to process visual and auditory information called the "dual channel" (cf. Paivio, 1986); and 3) the fact that learning is an active process that involves a set of cognitive processes that occur during learning. A set of principles, deriving from this theory, help us to design more efficient digital educational resources. Among them: the coherence principle implies that in a multimedia message the words, the images and the sounds that are irrelevant for learning should be eliminated, the temporal and space contiguity principles state that it is preferable to present text and images simultaneously; and the modality principle states that in a multimedia presentation the words should be narrated rather than written to avoid overloading the visual and spatial channels.

\section{Overview of the 4C/ID-model}

The central message of the 4C-ID-model and also of this paper is that settings for complex learning ${ }^{1}$ can always be described by four interrelated blueprint components: 1) learning tasks: authentic whole-tasks aiming towards the integration of skills, knowledge and attitudes. The set of learning tasks exhibits high variability and is organized into easy-to-difficult tasks and diminishing the support given to learners throughout each class task; 2) Supportive information (the theory): information helpful for learning and performing the problem-solving and reasoning aspects of the learning tasks. This information provides a bridge between what the learners already know and what they need to know to fruitfully work on the learning tasks; 3) procedural information (the how to's): information that is a prerequisite for learning and performing routine aspects of learning tasks. This information is displayed only at the moment it is needed, and for this reason it is also referred to as 'just-in-time' information (JIT); and 4) part-task practice: practice items provided to learners to help them reach a very high level of automaticity for selected routine aspects of a task.

Complex learning is always involved when a learner wants to acquire integrated sets of learning goals. The aim of complex learning is the integration of knowledge, skills and attitudes in one rich, interconnected knowledge base. So, the learning task should confront the learner with all or almost all of the constituent skills that are relevant to performing the learning tasks and at the same time with the associated knowledge and attitudes. Constituent skills often need to be controlled by higher-level strategies because they make little sense without taking their related constituent skills and associated knowledge and attitudes into account. For this reason, constituent skills are seen as aspects rather than parts of a complex skill. In a whole-task approach, learners are directly confronted with many different constituent skills from the start of training, although they cannot be expected to independently coordinate all those aspects at that moment. Thus, it is necessary to simplify the tasks and to give learners support and guidance, which are the supportive and procedure information function. A well-designed training blueprint ensures that learners are not overwhelmed with the complexity of a task, because tasks are ordered from easy-to-difficult, support and guidance are given when needed, and different types of information are presented precisely at the right time.

\footnotetext{
${ }^{1}$ Complex learning involves the integration of knowledge, skills and attitudes.
} 
Learners should not invest all their cognitive resources into performing the task, but should invest sufficient mental effort into genuine learning, that is, schema construction. This category of learning is responsible for the (i) construction of cognitive schemas, which might be interpreted by a controlled process to generate behaviour in new, unfamiliar situations, and (ii) rule automation, corresponding to the learning process responsible for the automation of cognitive schemas, which contain cognitive rules that directly guide behaviour without the need for conscious control.

\section{Multimedia learning and the 4C/ID-model}

As discussed in the previous section, the aim of the four components is to facilitate the learning process, with clear implications for the selection of suitable educational media and relevant multimedia principles. So, linked to the four components there are 22 multimedia principles (Mayer, 2014): (i) six associated with the learning tasks in computer-simulated environments, virtual reality systems, serious games and highfidelity simulators (sequencing principle, physical-fidelity principle, training-wheels principle, variability principle, collaboration principle and completion-strategy principle); (ii) seven associated with the supportive information in hypermedia systems, micro worlds and social media (prior knowledge principle, multimedia principle, dynamic visualization principle, redundancy principle, coherence principle, selfexplanation principle, self-pacing principle); (iii) five associated with procedural information with mobile apps, augmented reality, on-line help systems and pedagogical agents (modality principle, temporal split-attention principle, spatial split-attention principle, signalling principle and segmentation principle); (iv) one associated with parttask practice in drill and practice computed-based training systems (component-fluency principle); and (v) three related to the instructional control with adaptive systems and electronic development portfolios (individualization principle, second-order scaffolding principle and development portfolio principle).

Designing, developing, implementing and evaluating a learning environment according to this model is not an easy task and it requires various types of conceptual and technical knowledge. However, there is some empirical evidence on the effectiveness of the 4C/ID-model on complex learning training programs such as medicine and dental medicine (Postma \& White, 2015, 2016; Vandewaetere, et al., 2015; Maggio, et al., 2015), and science and informatics (Lim, et al., 2009; van Rosmalen, et al., 2014; Melo \& Miranda, 2015; Halil \& Elkhider, 2016).

Recent instructional models, such as the 4C/ID-model, stress the use of whole-tasks as a starting point to the learning process. But, there is a severe risk on the use of this kind of strategy because of the implicit task complexity. Cognitive load theory offers guidelines to deal with the very limited processing capacity of the human working memory.

Due to the task complexity of the whole-task practices, students may have difficulties with learning. In the 4C/ID approach, it is important to be careful with cognitive load management. According to Sweller (1988) there are three different types of cognitive load that need to be considered by instructional designers: 
1) Intrinsic cognitive load that is a direct function of performing the task, in particular, the number of elements that must be simultaneously processed in the working memory.

2) Extraneous cognitive load results from the ineffective instructional design and needs to be prevented.

3) Germane cognitive load imposed by processes directly relevant for learning (schema construction and automation). A well-designed instruction should optimize germane cognitive load within the limits of the total available working-memory capacity.

A basic assumption of the cognitive load theory is that an instructional design that results in a good use of the working memory capacity, due to low extraneous cognitive load induced by appropriate instructional procedures, may be further improved by encouraging learners to engage in conscious cognitive processing directly relevant to learning. Intrinsic, extraneous and germane cognitive load are additive so the total of the three types of cognitive load cannot exceed the working memory capacity. Consequently, the greater amount of germane cognitive load created by the instructional design will increase the learning potential.

How can you manage these three types of cognitive load? The 4C/ID-model suggests the following strategies, in terms of task sequencing and information presentation, to manage these types of cognitive load: (i) sequence learning task classes from simple-tocomplex. In order to decrease the cognitive load induced by complex tasks, the first task class should be the simplest version of the whole-task that learners encounter in the real world. For learning tasks within an easier task class, fewer elements and interactions between elements need to be processed simultaneously in working memory, so as the task class becomes more complex, the number of elements and interactions between the elements increases, for example, in the analysis of an electrical circuit it is relevant to present to the students the circuit diagram, so an increase in complexity corresponds to the introduction of more elements in the circuit connected in different forms, which leads the students to train the competence "analysis of a circuit"; (ii) sequence learning tasks with decreasing learner support (fading from high-to-no support) is another way of managing learners' cognitive overload imposed by complex whole tasks. Cognitive load theorists have developed alternative formats for learning tasks to adjust learner activities in a way that reduces the extraneous cognitive load caused by conventional tasks. Van Gerven et al., (2002) verified that worked examples facilitate schema construction and transfer performance more than actually solving the equivalent problems do. Other researchers suggest the use of completion tasks, with an intermediated level of support, combining the advantage of worked examples and conventional learning tasks (cf. Paas, 1992; van Merriënboer \& de Crook, 1992); (iii) sequence learning tasks in a variable order (high contextual interference). In order to increase germane cognitive load and thus facilitating learning, each learning task should be sequenced in a highly variable order, that is, each subsequent learning task is different from the previous ones in terms of dimensions; the reason for sequencing learning tasks in a variable order is because this increases the chances that similar features can be identified and that relevant features can be distinguished from irrelevant ones. Variability of practice almost affects the development of schemata and promotes subsequent learning transfer by an inductive process; (iv) present supportive information before learners start working on the 
learning tasks and make it accessible to learners during the whole-task practices, because supportive information involves mental models (e.g. "What is this?"; "How is this organized?"; "How does this work?") and cognitive strategies (e.g. heuristics to solve problems) with high inherent complexity; (v) Present just-in-time information when learners need it to prevent temporal and spatial split-attention effects; and finally (vi) part-task practice automates particular recurrent aspects of the complex skill, because automated recurrent skills may decrease the cognitive load associated with performing the whole-task, making performance of the whole skill more fluid, decreasing the chance of making errors due to cognitive overload.

The 4C/ID model suggests 10 steps for a detailed training blue print: 1) decompose the complex skill; 2) sequence task classes; 3) design learning tasks; 4) analyse mental models; 5) analyse cognitive strategies; 6) design supportive information; 7) analyse rules and procedures; 8) analyse prerequisite knowledge; 9) design just-in-time information; 10) design part-task practice. Four of those steps $(3,6,9$ and 10) pertain, in order, to the four components of the 4C/ID-model. The remaining six steps have an analytical nature and allow for the effective design of learning activities. For example, in step 1 (decompose the complex skill), first the whole complex skill is deconstructed into its constituent skills and the appropriate performance objectives are formulated for each constituent skill; according to the characteristics of the desired final performance objectives the constituent skills are classified as either recurrent (routine) our non-recurrent skills (varies from problem to problem). Step 2 (sequence task classes), yields the first outline of the training program, steps 4 (analyse mental models) and 5 (analyse cognitive strategies) are focused on the analysis and identification of general knowledge required for students to perform non- recurring aspects of the skill.

\section{LEARNING ENVIRONMENT DESIGN}

This section describes the methodology used to design a digital learning environment to teach $9^{\text {th }}$ grade students to analyse a simple electrical circuit (called "Circuitos Elétricos"). All the steps followed to produce the final version of the prototype are described. The digital educational resource was built with Adobe Flash ${ }^{\circledR}$ CS3 Professional and it works both in MacOS and Windows environments.

\section{Set performance objectives}

The instructions for the digital learning resource design follow the principles of the 4C/ID-model (van Merriënboer, 1997). The design started by skill analysis and decomposition. Skill decomposition, splitting a skill into all of its components or basic elements, aims at the description of all constituent skills and interrelationships between them, which together make up the complex cognitive skill. The result of this skill decomposition process is a skill hierarchy that is represented in the design blueprint shown in Figure 1. 


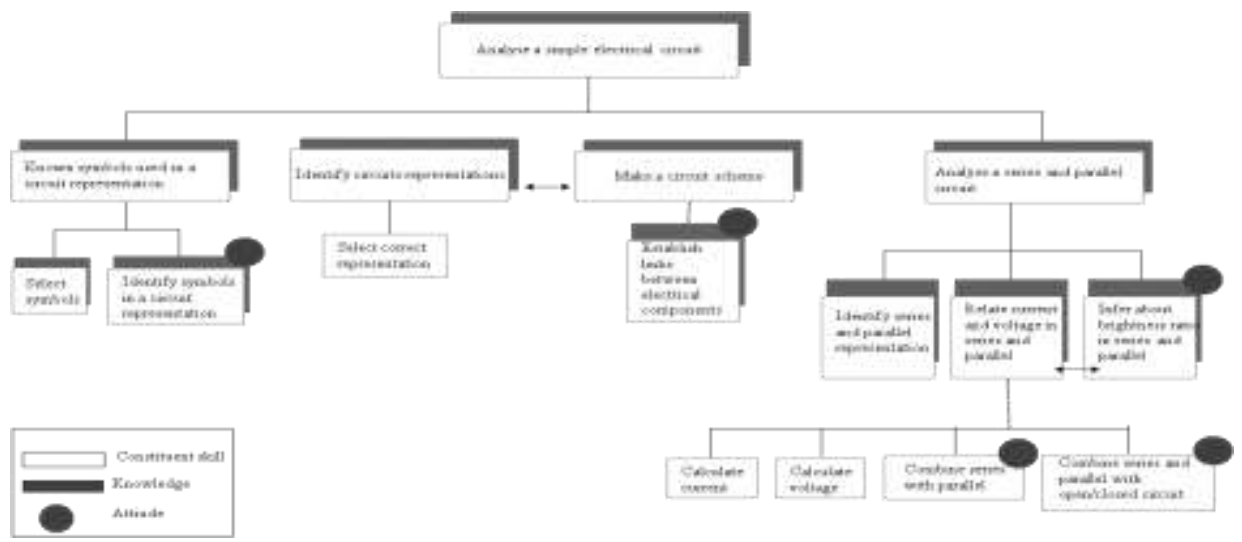

Figure 1

A hierarchy of constituent skills with an indication of associated knowledge and attitudes for the complex skill "Analyse a simple electrical circuit".

A well-designed training program for $9^{\text {th }}$ grade physics students will not teach each of these constituent skills separately, but will teach them in an integrated fashion. As can be seen from Figure 1, a hierarchy of constituent skills is used as an organizing framework for the whole knowledge base. Knowledge and attitudes are fully integrated in this framework, subordinate to the constituent skills. Constituent skills adjacent to each other horizontally can be performed sequentially (e.g., you first have to "Know symbols used in a circuit representation", then "Identify circuit representation", then "Make a circuit diagram" and then "Analyse a series and parallel circuit") or simultaneously (e.g., you first have to "Know symbols used in a circuit representation", then "Identify a circuit representation" and simultaneously "Make circuit diagram" and then "Analyse a series and parallel circuit"). Constituent skills at a lower level, on the vertical dimension, enable the learning and performance of skills higher in the hierarchy (e.g., you must be able to "Select symbols" to "Identify symbols in a circuit representation"; you must be able to "Establish links between electrical components" to "Make a circuit diagram"; you must be able to "Identify series and parallel representation", to "Relate current and voltage in series and parallel" and to "Infer about brightness ratio in series and parallel" in order to be able to "Analyse a series and parallel circuit").

\section{Design learning tasks}

The application "Circuitos elétricos" is structured in three lessons: lesson 1, lesson 2, and lesson 3. Lesson 1 is made up of three learning tasks and has two main objectives: 1) to introduce the concept of electric current and electrical conductivity and 2) to allow students to have a first contact with the learning environment, thus helping them to reduce the cognitive load associated with routine skills, like knowing how to access supportive information and the sequence of the learning tasks. All learning tasks were organized according to the sequencing principle, i.e., from simple-to-complex. 
In lesson 2 the symbols of the devices used in the representation of an electrical circuit are introduced. In this lesson students have to associate the device name with its symbol. The devices are always presented in a circuit diagram, because the competence to be worked on is the analysis of a simple electrical circuit. Figure 2 (a) shows a circuit used in this lesson for students to name the devices shown.

In lesson 3 the main objective is to introduce the concepts of series and parallel circuits. In this lesson students are able to relate to a series and parallel circuit: bulb brightness, voltage and current. All learning tasks are presented via a simple electrical circuit analysis approach. Figure 2 (b) represents a series circuit in which students are expected to draw connections between voltage and current.

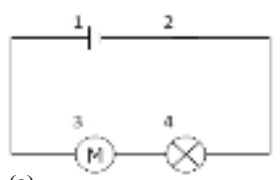

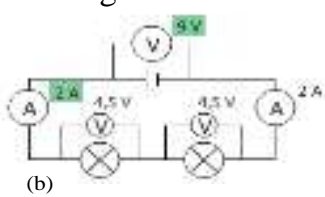

(b)

Figure 2

(a) Example of a circuit for lesson 2 and (b) example of a circuit for lesson 3.

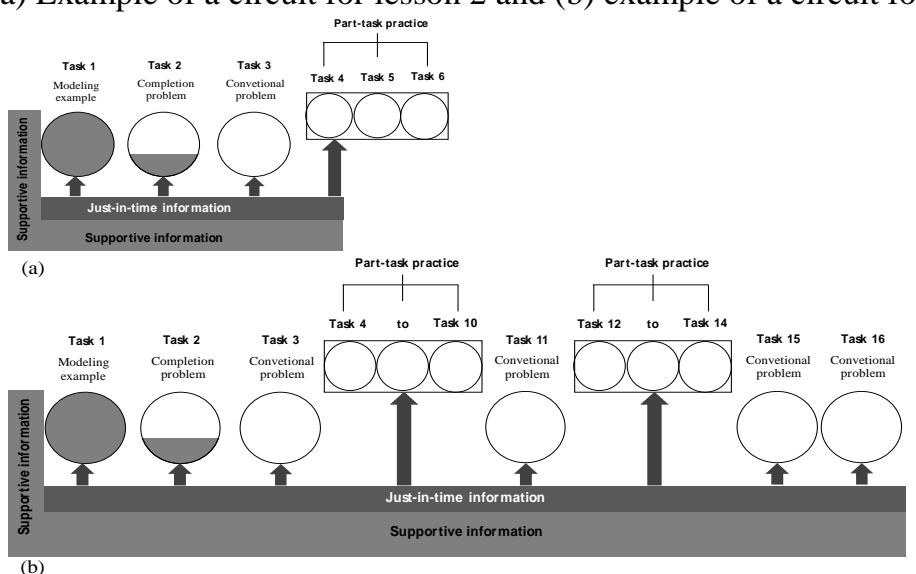

Figure 3

Sequence of learning tasks in lessons 2 (a) and 3 (b).

Figures 3 (a) and (b) show the sequences of the learning tasks for lessons 2 and 3 . The circles represent the learning tasks and the gray fill represents the level of support and guidance. Students were presented with a modeling example ${ }^{2}$ with two parts: first students watched a video with an explanation of the theoretical concepts and then they were required to follow all the steps of a worked problem, always receiving messages that explained the decision making process at each step. In this case constituent skills

\footnotetext{
${ }^{2}$ Modeling examples are problems in which an expert demonstrates why he or she is doing what he or she is doing in order to reach a solution (van Merriënboer, 1997).
} 
are taught in the context of the whole-task and not as isolated procedures as in a parttask approach. According to the 4C/ID-model principles, this modeling example was the first whole learning task that learners encountered (training-wheels principle).

After completing the first learning task, the students received a second whole learning task - a completion problem, as a practice activity (completion-strategy principle). And then students are confronted with a conventional problem ${ }^{3}$ without support and guidance.

In lesson 2 the students are put in contact with the symbology used in a circuit schema as well as with simple circuit diagrams with multiple components. Task 1 corresponds to a worked example (modelling example) in which students had to click on the icon on which the name of the electronic device is inscribed so that the appropriate symbol representation emerges. Modelling examples provide maximum guidance, because learners are confronted with a task similar to the whole-tasks that need to be studied and evaluated by the learner, in which the learner pays explicit attention to the solving process.

Task 2 corresponds to a completion problem in which students had to choose the option that matches the names of the three devices that do not have the name inscribed. Finally, in task 3, learners are confronted with a conventional problem in which they had to select the option that matches the names of the five devices shown in each white square. Tasks 4, 5 and 6 correspond to practical exercises in which students had to identify the symbols of electric devices in simple electrical circuit representation.

In lesson 3 learners are exposed to series and parallel circuits. Task 1 corresponds to a worked example (modelling example). This is a whole-problem in which all features that aim to teach about series and parallel circuits are shown. In this example the students are required to analyse a circuit diagram and to read all the questions and their answers in order to advance to the next item. Therefore, the task shows the general model used in the analysis of this kind of circuit.

Completion tasks provide learners with a given state, criteria for an acceptable goal state and a partial solution. The leaners must complete the partial solution by determining and adding the missing steps, either at the end of the solution or in one or more places in the middle of the solution. A particularly strong point of completion tasks is that the learners must carefully study the partial solution provided to them, because otherwise they will not be able to come up with the complete solution. Van Merriënboer and de Crook (1992) suggest that completion tasks are especially useful in design-oriented task domains and were originally developed in the domain of science and software development, where learners had to fill in missing command-lines in partial computer programs. A well-designed completion task ensures that learners can understand the partial solution and still have to perform a non-trivial completion.

\footnotetext{
${ }^{3}$ Conventional problems are problems for which some given state and a specific goal state are provided and the task is to find a solution to reach the goal (van Merriënboer, 1997).
} 
The conventional tasks used in this learning environment are, according to van Merriënboer and Kirschner (2009), called imitation tasks in which learners are confronted with a conventional task in combination with a case study of an analogous task. So, the solution presented in the case study provides a blueprint for approaching the new task, focusing attention on possibly useful solution steps. The required imitation is a sophisticated cognitive process where learners must identify the analogy between the case study and the given task and use the case study to plan a new solution (Vosniadou \& Ortony, 1989). Imitation tasks are quite authentic, because experts often rely on their knowledge of specific cases to guide their problem solving behaviour on new problems (case-based reasoning).

The common element of all of these types of learning tasks is that they direct the learners' attention to problem states, acceptable solutions and useful solution steps. This helps them mindfully abstract information from good solutions or use inductive processes to construct cognitive schemas that reflect generalized solutions for particular types of tasks. This design was chosen because for novice learners, studying useful solutions together with the relationships between the characteristics of a given situation and the solution steps applied, it is much more important for developing problem solving and reasoning skills than solving equivalent problems (van Merriënboer \& Sweller, 2005). Only more experienced learners, who have already developed most of the cognitive schemas necessary to guide their problem solving, should use conventional tasks without support and guidance. This is why it was decided to introduce a series of learning tasks in lessons 2 and 3 to train and stimulate the development of cognitive schemes for the resolution of conventional tasks that are closer to real-world tasks.

All learning tasks were designed with care to eliminate irrelevant aspects for the training program, as required by the physical-fidelity principle. In each learning class it was decided to diversify the types of items, such as true and false items, multiple choice items, completion items and drag-and-drop items (variability principle).

\section{Design supportive information}

Having designed the learning tasks, the next step is to design supportive information to carry out the learning tasks. It is this information that bridges the gap between the knowledge of the students and what they need to know to develop the complex skills they are learning. Supportive information helps learners to establish meaningful relationships between newly presented elements and their prior knowledge. This process yields rich cognitive schemas that relate many elements to many other elements. Such schemas allow for deep understanding and increase the availability and accessibility of task related knowledge in long-term memory.

Supportive information is the information that teachers typically called "the theory" and it is divided into three parts: 1) description of the domain models ${ }^{4} ; 2$ ) description of the

\footnotetext{
${ }^{4}$ A description of learning in terms of applicable facts, concepts, principles and plans. It is the result of the analysis of metal models. Examples of domain models are conceptual models, causal models and structural models (van Merriënboer \& Kirschner, 2009).
} 
systematic approaches to problem solving $-\mathrm{SAPs}^{5}$; and 3) the cognitive feedback ${ }^{6}$ linked to the performance quality. In this project the domain models were presented in the form of videos (called "Informação de suporte"/"supportive information"), the cognitive strategies through a worked-example and the cognitive feedback with multimedia messages that arise when the students select their response. The design of supportive information videos followed the principles of the multimedia learning theory referred to in Multimedia learning and the 4C/ID-model section, mainly, the absence of sounds, words and images that are not relevant for learning, the simultaneous presentation of text and images, and the presentation of images (statics and moving) with narrated words.

Supportive information reflects the mental models (domain models) that allow us to reason within the task domain. There are four types of models: (i) conceptual models, which correspond to the concepts and their elements allowing the description and classification of objects or tasks (helping the learner to answer to the question "What is this?"); (ii) structural models, which describe how objects or tasks are related to each other in time and space (it's related to organization); (iii) casual models that focus on how objects or tasks affect each other and help the learner to interpret processes, give explanations and make predictions (such models help the leaners to answer the question "How does this work?"); and (iv) causal models that relates an action to an effect, called principles; so causal models deal with principles or theories. In this case it was presented the domain model as a conceptual model in lesson 2, because typical kinds of electrical circuits were discussed, as well as the state of typical components such as switches, resistors and batteries. On the other hand, the domain model presented in lesson 3 was a causal model, because it was presented principles of series and parallel circuits that allow the learners to draw implications by predicting a certain phenomenon such as the current and voltage in circuit branches.

Supportive information also reflects knowledge with respect to cognitive strategies that allow us to perform tasks and to solve problems (SAPs); it specifies the phases that an expert typically goes through while carrying out the task as well as the rules-of-thumb that may be helpful to successfully complete each phase. In this case this kind of knowledge is presented to the students in the worked example that appears at the beginning of each lesson.

What is the best strategy to present the supporting information? There are three strategies: 1) deductive strategy; 2) inductive strategy; and 3) guided discovery. In this case it was decided for the deductive strategy because it is the most common strategy that allows us to manage time in a more effective way, and it is more suitable for the participants' age. Deductive presentation strategy works from general and abstract

\footnotetext{
${ }^{5}$ A description of a systematic approach to problem solving in terms of subsequent problemsolving phases and rules-of-thumbs that may help to successfully complete each phase. It is the result of an analysis of cognitive strategies (van Merriënboer \& Kirschner, 2009).

${ }^{6}$ The type of feedback that allows the learner to reflect on the quality of found solutions or the quality of the problem solving process (van Merriënboer \& Kirschner, 2009).
} 
information presented in SAPs and domain models toward concrete illustrations of this information. In this case, the first learning tasks of each lesson took the form of a modeling example that was used to illustrate the presented SAPs and domain model. For instance, in lesson 3 students had to recognize series and parallel circuit representation so, general information included the differences between the two types of circuits and the first learning task was a worked-example that explained the problem-solving phases and rules-of-thumb that may help learners to successfully complete the task. In our project the "theory" is first presented, and after students should "apply" this theory to the first learning task that is a worked-example.

Cognitive feedback consists of information (including prompts, cues and questions) that helps learners to construct or reconstruct their cognitive schemas in such a way that future performance is improved. Cognitive feedback stimulates learners to reflect on the quality of both their personal problem-solving processes and the solutions that they have found, thus more effective cognitive strategies and mental models can be developed. This kind of feedback is provided after learners have completed a learning task. For example, in this learning environment students should select the correct values marked by ammeters $\mathrm{X}$ and $\mathrm{Y}$ and if they clicked the wrong alternative, an error message appeared (in the blue box) with the following information "Wrong answer! See how the lamps are associated." So, the main idea of this learning task is to determine the current flowing through each lamp, but first the students should recognize how the lamps are connected.

\section{Design procedural information}

Procedural information refers to: 1) just-in-time (JIT) information displays, providing learners with the rules or procedures that describe the performance if recurrent aspects of a complex skill are used and if knowledge prerequisite is required for correctly carrying out those rules or procedures; 2) demonstrations of the application of those rules and procedures as well as instances of the prerequisite knowledge; and 3) JIT of corrective feedback errors. Considering the participants age and the kind of content taught, procedural information is focused only on corrective feedback.

In contrast to cognitive feedback, the main function of corrective feedback is not to foster reflection, but rather to detect and correct errors that involve a direct modification of the learner's actions (Argyris \& Schön, 1978), for example, in a learning task in which students have to select the correct option, if they select the incorrect option a corrective feedback message appears with the following information "Wrong answer! Look carefully at the switches' positions", for students to re-analyze the circuit. This kind of feedback message is presented not only to help learners to recognize that an error has been made, but also to explain why there is an error, giving them a hint as to how to reach the desired goal. This feedback should promote the compilation of newly acquired knowledge into cognitive rules. After the knowledge is compiled, the solution is generated by these new rules. In this case, students were expected to identify, from a description of the diagram of an electrical circuit, that the complex learning is related to the generation of the circuit image, combining the features of each component on the circuit. Therefore, it is necessary that the students compile the rules inherent to such representation, in a similar way to what a computer does when compiling a program. 


\section{Design part-task practice}

Part-task practice should always be provided in the context that learners must already be able to relate and integrate them into required whole-task performance. This could be reached by first presenting modelling examples or other learning tasks (completion examples) that allow learners to understand how the part-task fits into the whole-task. Were proposed two sets of practical problem after learners have analysed a modelling example: a completion problem and solving a conventional problem. So these sets of part-task problems are inserted into a fruitful cognitive context, because learners are able to relate and integrate knowledge, attitudes and skills that are necessary to analyse a simple electrical circuit. The second important aspect is the distribution of the part-task training over time. Van Merriënboer and Kirschner (2009) recommend that it is best to distribute sessions over time, alternating with learners working on learning tasks, and Schneider (1985) mentions that this intermixing procedure promotes integration of knowledge, attitudes and skills.

\section{METHOD}

\section{Participants}

The research was carried out in a private school in Lisbon, using a sample of 125 students from the $9^{\text {th }}$ grade (age: $\mathrm{M}=14.3$ years; $\mathrm{SD}=0.54$ ), distributed throughout five classes. Although each class was a natural group (without random selection), the selection process of the groups for the experimental $\left(n_{E}=76\right)$ and control $\left(n_{C}=49\right)$ groups was random.

\section{Instruments}

To study the effect of the 4C/ID approach, two achievement tests were used: (1) The reproduction test that consists of 14 multiple-choice items (each correct answer is 1 point and each wrong answer is 0 points) that were similar to the learning tasks carried out by students in both groups, (2) a multiple-choice learning transfer test, applied to both groups, consisting of 14 items (each correct answer is 1 point and each wrong answer is 0 points), which appealed to the application of acquired knowledge to different situations. The reproduction test used the same circuit diagrams that were worked out in the classes and the transfer test, the circuit diagrams were used in the different contexts, such as the presentation of the devices in different positions in the circuit, more elements in series and parallel and the use of lamps with different resistances.

The reactions and behaviours of both groups of students were also observed at the beginning and during the sessions in which the experiment took place and at the end of the training programme, the teachers who were involved in this project were interviewed. Thus, an observation sheet and an interview guide were created to collect the qualitative data.

\section{Procedure}

In the experimental group the theme "Electrical Circuits" was taught through a digital learning environment based on the 4C/ID-model. This environment was composed of three sessions (learning classes) with distinct objectives: Class 1 - exploration of the 
concepts of electric current and voltage with the intention of promoting the first contact with the features learning environment; Class 2 - learn how to create a simple electrical circuit; and Class 3 - learn the characteristics of a series and parallel association. In the control group the same subject was taught based on a conventional method (teachercentred with a daily notebook, a student manual and a simple calculator). Three teachers were involved in this research: one teacher (the researcher) with one class from the experimental group and two more teachers, each one with one class from de experimental group and another class from the control group. The two groups performed similar learning activities.

The achievement tests were applied to both groups in two moments: in the last class of the training programme ( $1^{\text {st }}$ application) and one week after the training programme (follow-up).

The experiment was conducted as follows: the classes of the two groups started to study the electrical circuits on the same day using different instructional methods. The experimental groups used a computer room with one computer for per student and with an individual audio system to carry out the learning tasks proposed in the digital educational resource. The control group had classes in their classroom and used the resources provided by the teachers (PowerPoint presentations, simulations explored by the teachers, a manual, a notebook, a pencil and a calculator). The experiment was conducted in two 90-minute sessions over two weeks.

Three teachers were involved. One of the teachers who was the researcher stayed with an experimental group class and the other teachers had an experimental class and a control class each.

\section{FINDINGS}

Before presenting the results, it is important to highlight that the main objective of this study is to describe how the digital resource was thought up and constructed. This justifies the option to present the results referring to student performance on the topic of a simple electrical circuit analysis with a brief description of the students' reactions and behaviour and teacher perception about the experience. Other studies have covered different variables such as the perceived mental effort of students in achievement tests, the instructional efficiency (Melo \& Miranda, 2015) and students approaches to learning (Melo \& Miranda, in press).

The mean value of the scores obtained in each of the tests for the $1^{\text {st }}$ application and for the follow-up of both groups were presented. The graphical representation of each group's performance in both tests was shown in Figure 4.

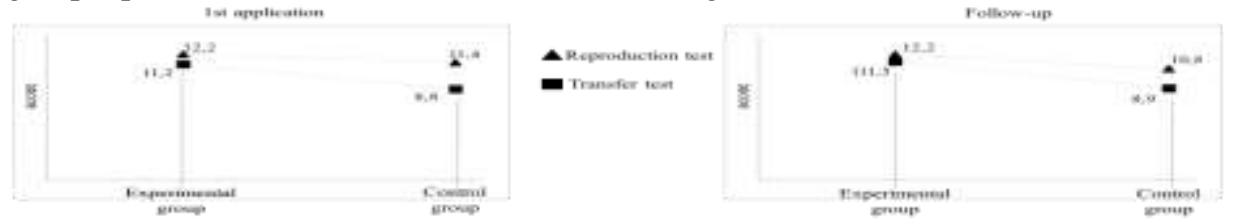

Figure 4

Performance of both groups on the reproduction and transfer test for the $1^{\text {st }}$ application and for the follow-up application. 
As shown in Figure 4, students from the experimental group obtained similar scores on both reproduction and transfer tests and students from the control group obtained lower results in both achievement tests. Observing the performance of the two groups in both tests, it is found that the students in the control group tended to score slightly lower than those of the students in the experimental group in the reproduction test. However, with respect to the transfer test scores, the results show that the students in the control group have much lower scores when compared with those in the experimental group.

In order to quantify these differences, was calculated the percentage difference from the experimental group using the formula:

$\%$ difference $=\frac{\mathrm{M}_{\mathrm{EG}}-\mathrm{M}_{\mathrm{CG}}}{\mathrm{M}_{\mathrm{EG}}} \times 100$

$\mathrm{M}_{\mathrm{EG}}$ and $\mathrm{M}_{\mathrm{CG}}$ are the mean scores of the achievement tests for the experimental group and the control group respectively. The following values were collected: for the $1^{\text {st }}$ application $6.6 \%$ for the reproduction test and $21.4 \%$ for the transfer test; for the followup application $11.4 \%$ for the reproduction test and $22.6 \%$ for the transfer test. Then, it turns out that students from the experimental group performed better in the application of knowledge about electrical circuits in new problem situations.

In order to interpret the differences, found on achievement tests scores it was also relevant characterizing the reactions and behaviours of the students of the two groups during the training sessions. Thus, the two sessions of the two groups were observed and the reactions and behaviours of the students recorded at the beginning and during each session. Interviews to teachers were conducted to get their perceptions about students' reactions and behaviours.

Table 1 presents the results of observation of the reactions and behaviours of the students of the two groups throughout the two training sessions.

Table 1

Summary of reactions and behaviours of students in both groups.

\begin{tabular}{|c|c|c|}
\hline & Experimental group & Control group \\
\hline $\begin{array}{l}\text { At the } \\
\text { beginning ... }\end{array}$ & $\begin{array}{l}\text { The students were very } \\
\text { curious and agitated about } \\
\text { being in a different class ( } 1^{\text {st }} \\
\text { session). }\end{array}$ & $\begin{array}{l}\text { Students entered the classroom } \\
\left.\text { normally ( } 1^{\text {st }} \text { and } 2^{\text {nd }} \text { sessions }\right) .\end{array}$ \\
\hline $\begin{array}{l}\text { During the } \\
\text { class ... }\end{array}$ & $\begin{array}{l}\text { - The students were very } \\
\text { involved and focused on the } \\
\text { tasks ( } 1^{\text {st }} \text { and } 2^{\text {nd }} \text { sessions); } \\
\text { - The students took notes } \\
\text { autonomously ( } 1^{\text {st }} \text { and } 2^{\text {nd }} \\
\text { sessions); } \\
\text { - Students completed all tasks } \\
\left(1^{\text {st }} \text { and } 2^{\text {nd }} \text { sessions }\right) \text {. }\end{array}$ & $\begin{array}{l}\text { - The students were attentive to } \\
\text { the teacher's exposition ( } 1^{\text {st }} \text { and } \\
2^{\text {nd }} \text { sessions); } \\
\text { - The students showed little } \\
\text { involvement and focus on the } \\
\text { tasks ( } 1^{\text {st }} \text { and } 2^{\text {nd }} \text { sessions); } \\
\text { - Some students did not complete } \\
\text { all tasks }\left(1^{\text {st }} \text { and } 2^{\text {nd }} \text { sessions }\right) \text {. }\end{array}$ \\
\hline
\end{tabular}


The interviewed teachers reported that during the completion of the learning tasks the students of the experimental group were very focused and committed, always showing great care when reading the messages of the corrective and cognitive feedback system. The fact that the students could manage their learning rhythm was mentioned as very positive. Regarding the control group students, the teachers reported that during the writing tasks the students were more dispersed and more distracted. It was also mentioned that some students in this group could not finish their learning tasks autonomously because the learning rhythm was totally teacher-centered.

The students in the control group completed almost all of the short answer tasks that involved the knowledge reproduction; however the tasks that involved the learning transfer were incomplete or not performed. It was also found that the students in the control group had greater difficulty in performing the tasks that involves the analysis of series and parallel circuits, because they imply the establishment of more relationship between physical variables (e.g. electric voltage, electric current and electric resistance) which implies a must more focussed attitude.

\section{DISCUSSION AND CONCLUSION}

The main purpose of this study was to introduce a framework to design a digital learning environment with the 4C/ID-model and to analyse a learning example to teach electrical circuits to students of the $9^{\text {th }}$ grade of compulsory education. It was also our objective to study the effects of the instructional approach (4C/ID versus conventional) on the students' learning, in particular on students' knowledge reproduction and learning transfer.

The main compelling reason for using the approach of complex learning in physics education, is that the whole-task models (like the 4C/ID-model) provide a solid framework for the development of learning activities that foster students' functioning in a varied set of complex learning situations. As a result of using this kind of approach, the development of a rich set of mental models and cognitive strategies is facilitated, allowing students to use efficient problem-solving strategies in electrical circuits. By using authentic tasks, complex learning is supported and hence the transfer from the digital environment to another situation becomes more feasible. That's why the control group students had the lower scores in the transfer test.

Through quantitative data on students' performance and qualitative data, it is relevant to highlight the positive findings of this experiment: 1) the fact that the learning tasks were in a computerized environment, in which students worked individually, which made the learning process totally centred on students, leading them to an autonomous and efficient management of learning rhythm; 2) corrective and cognitive feedback systems stimulated the transfer of learning and allowed the establishment of relationship between students' prior knowledge and new knowledge, promoting the construction and the improvement of mental frameworks; the efficient management of working memory resources making them more available for analysis and reflection on the contents of instructional materials; 3 ) the use of new cognitive learning theories made the design of 
learning resources more attractive and efficient for students and gave them a more active role in the learning process.

Differences found on scores obtained by the two groups in the learning transfer test can be interpreted based on the theoretical framework and from the qualitative data. These differences can be explained by a combination of two factors: 1) the 4C/ID-model is based on the principles of cognitive load theory, so the interaction between the four components allows the minimization of the cognitive load imposed by the instruction, which probably implies that the students of the experimental group perceived less cognitive load in the application of learned knowledge to new situations and (2) qualitative data shows that students in the experimental group solved learning tasks on electrical circuits with greater concentration and commitment when compared to the control group, which may also have occurred in the performance of the learning transfer test.

In conclusion, there are 10 steps to follow for the development of whole-tasks based on the 4C/ID-model. These steps offer science teachers and instructional designers a way to introduce further the whole-task approach in physics education and to deal with practical, theoretical and technical issues. In addition, new research paths can be developed using the whole-task approach, i.e., this digital learning environment could be tested on other secondary schools and other physics themes can be learned using this kind of instructional approach.

It is important to point out that the results presented in this article are part of other studies and they were already mentioned, the main purpose of this paper is to describe the method used in the design and construction of an educational resource based on the 4C/ID-model. It should also be noted that the results presented here are only valid for this population, which suggests that the use of the 4C/ID-model in the classroom context seemed to be more efficient than a conventional teaching method. Thus, this study intends to provide a contribution to the validation of the use of the 4C/ID-model in the classroom for this age range.

As already mentioned, Melo and Miranda (2015) studied the instructional efficiency of this learning environment with a group of 129 students from the $9^{\text {th }}$ grade. The main objective of the study was to investigate the effect of the "circuitos elétricos" digital environment on the students' performance variables (reproduction and learning transfer) and on the perceived mental effort (cognitive load measurement) by the students, to study the effect of the instructional approach on the instructional efficiency. The authors perform a quasi-experimental study comparing two different instructional approaches: the 4C/ID-model (experimental group, $\mathrm{n}=78$ ) versus a more traditional approach (control group, $\mathrm{n}=51$ ) based on a teacher-centred strategy. The results revealed: 1) significant differences in the level of performance (reproduction and learning transfer) of the students, and these differences were greater in the learning transfer; 2) a more marked decrease in the perceived mental effort by the students in the learning transfer tests. In general, the results showed that the use of the digital learning environment led students to solve learning tasks more efficiently, Therefore, students who used the 
digital learning environment developed mental schemes that allowed them to apply the acquired knowledge to new situations with a lesser perceived mental effort.

In another study (Melo \& Miranda, in press), the same authors found that the use of the 4C/ID approach modified the type of students learning approach, verifying that students who use this type of instructional approach tended to adopt a deeper learning approach.

\section{SUGGESTIONS FOR FUTHER RESEARCH}

The main limitation of this study was the deficit of variables studied that allowed for the characterizing of the learning process in both groups. Thus, the study of the variables self-autonomy, motivation for learning and learning ability is recommended. So, it would be very important to combine quantitative data with qualitative data, namely through the use of research interviews and observation of how students solve problems. The use of a mixed-method research allows a deeper interpretation of qualitative data along with the frequency of quantitative results, in particular to better understand how students do transfer learning.

It would also be important to replicate this study more times over time to confirm the results of the 4C/ID-model's learning advantages.

The replication of this study with different samples of the same level of education is also important, because it is possible to understand the effect of the scholar population type, e.g., the socioeconomic level on learning process, which may affect the efficiency of the instructional model. It will also be interesting to see if the efficiency of the 4C/ID-model is affected by the subject/discipline in which it is applied at the same level of education.

\section{REFERENCES}

Argyris, C., \& Schon, D. (1978). Organizational learning: A theory of action perspective. Reading. Addison Wesly, MA.

Halil, M. K., \& Elkhider, I. A. (2016). Applying learning theories and instructional design models for effective instruction. Advances In Physiology Education, 40(2), 147. doi:10.1152/advan.00138.2015.

Kirschner, P. A. (2002). Cognitive load theory: Implications of cognitive load theory on the design of learning. Learning and Instruction, 12(1), 1-10.

Lim, J., Reiser, R., \& Olina, Z. (2009). The effects of part-task and whole-task instructional approaches on acquisition and transfer of a complex cognitive skill. Educational Technology Research \& Development, 57(1), 61-77. doi:10.1007/s11423-007-9085-y.

Maggio, L. A., Cate, O., Irby, D. M., \& O'Brien, B. C. (2015). Designing evidencebased medicine training to optimize the transfer of skills from the classroom to clinical practice: Applying the four component instructional design model. Academic Medicine, 90(11), 1457-1461. doi: 10.1097/ACM.0000000000000769. 
Mayer, R. E. (2014). The Cambridge handbook of multimedia learning(2nd Ed.). New York: Cambridge University Press.

Melo, M., \& Miranda, G. L. (2015). Learning electrical circuits: The effects of the 4CID instructional approach in the acquisition and transfer of knowledge. Journal of Information Technology Education: Research, 14, 313-337. http://www.jite.org/documents/Vol14/JITEv14ResearchP313-337Melo1752.pdf. Accessed 14 January 2017.

Melo, M., \& Miranda, G. L. (in press). Modelo 4C/ID: efeito sobre as abordagens à aprendizagem dos alunos do $9^{\circ}$ ano .Análise Psicológica.

Merrill, M. D., Drake, L., Lacy, M. J., \& Pratt, J. (1996). Reclaiming instructional design. Educational Technology, 36(5), 5-7.

Paas, F. (1992). Training strategies for attaining transfer of problem-solving skills in statistics: A cognitive-load approach. Journal of Educational Psychology, 84, 429-434.

Paas, F. G., Tuovinen, J. E., Tabbers, H., \& Gerven, P. W. (2003). Cognitive load measurement as a mean to advance cognitive load theory. Educational Psychologist, $38(1), 63-71$.

Paivio, A. (1986). Mental representation: A dual coding approach. New York: Oxford University Press.

Postma, T. C., \& White, J. G. (2016). Developing integrated clinical reasoning competencies in dental students using scaffolded case-based learning - empirical evidence. European Journal of Dental Education, 20(3), 180-188. doi:10.1111/eje.12159.

Postma, T. C., \& White, J. G. (2015). Developing clinical reasoning in the classroom analysis of the 4 C/ ID-model. European Journal of Dental Education, 19(2), 74-80. doi:10.1111/eje.12105.

Reiser, R. A., \& Dempsey, J. V. (2007). Trends and issues in instructional design and techonology (2nd ed). Merrill Prentice Hall, NJ.

Schneider, W. (1985). Training high-performance skills: Fallacies and guidelines. Human Factors, 27, 285-300.

Sweller, J. (1988). Cognitive load during problem solving: Effects on learning. Cognitive Science, 12, 257-285.

Sweller, J., Ayres, P., \& Kalyuga, S. (2011). Cognitive load theory. New York: Springer.

Van Gerven, P. W. M., Paas, F. G. W. C., van Merriënboer, J. J. G., \& Schmidt, H. G. (2002). Cognitive load theory and aging: Effects of worked examples on training efficiency. Learning and Instruction, 12, 87-105. 
Van Merriënboer, J. J. G. (1997). Training complex cognitive skills: A four-component instructional design model for technical training. Educational Technology Publications: NJ Englewood Cliffs.

Van Merriënboer, J. J. G., \& de Crook, M. B. M. (1992). Strategies for computer-based programing instruction: Program completion vs. program generation. Journal of Educational Computing Research, 8, 365-394.

Van Merriënboer, J. J. G., \& Sweller, J. (2005). Cognitive load theory and complex learning: recent developments and future directions. Educational Psychology Review, 17, 147-177. doi: 10.1007/s10648-005-3951-0.

Van Merriënboer, J. J. G., \& Kirschner, P. A. (2009). Ten steps to complex learning. Routledge, New York.

Van Merrienböer, J. J. G., \& Kester, L. (2014). The four-component instructional design model: Multimedia principles in environments for complex learning. In R. Mayer (Ed.). The Cambridge Handbook of Multimedia Learning (pp. 104-149). New York: Cambridge University Press.

Van Rosmalen, P., Boyle, E. A., Nadolski, R., van der Baaren, J., Fernández-Manjón, B., MacArthur, E., \& Star, K. (2014). Acquiring 21st Century Skills: Gaining Insight into the Design and Applicability of a Serious Game with 4C-ID. Games \& Learning Alliance, 327. doi:10.1007/978-3-319-12157-4_26.

Vandewaetere, M., Manhaeve, D., Aertgeerts, B., Clarebout, G., Van Merriënboer, J. G., \& Roex, A. (2015). 4C/ID in medical education: How to design an educational program based on whole-task learning: AMEE Guide No. 93. Medical Teacher, 37(1), 4-20. doi:10.3109/0142159X.2014.928407.

Vosniadou, S., \& Ortony, A. (1989). Similarity and analogical reasoning. New York, Cambridge University Press. 